www.jmscr.igmpublication.org

Impact Factor (SJIF): 6.379

Index Copernicus Value: 71.58

ISSN (e)-2347-176x ISSN (p) 2455-0450

crossref DOI: _https://dx.doi.org/10.18535/jmscr/v6i2.133

Journal Of Medical Science And Clinical Research

Original Research Article

\title{
A Comparative Study of Efficacy of Esmolol and Lignocaine for Attenuation of Stress Response during Laryngoscopy and Endotracheal Intubation in Normotensive Patients Undergoing General Anaesthesia
}

\author{
Authors \\ Dr Sonali Khobragade ${ }^{1}$, Dr Sandhya Manjrekar ${ }^{2}$, Dr Ramanand Jadhav ${ }^{3}$ \\ ${ }^{1}$ MBBS.MD (Anaesthesiology), Associate Professor, Dept. of Anaesthesiology, Indira Gandhi Government \\ Medical College and Hospital, Nagpur 440027, Maharashtra, India \\ ${ }^{2}$ MBBS.MD (Anaesthesiology), Professor and Head, Dept. of Anaesthesiology, Indira Gandhi Government \\ Medical College and Hospital, Nagpur 440027, Maharashtra, India \\ ${ }^{3}$ MBBS.DA (Anaesthesiology), Resident, Dept. of Anaesthesiology, Indira Gandhi Government Medical \\ College and Hospital, Nagpur440027, Maharashtra, India \\ Corresponding Author \\ Dr Sonali M. Khobragade \\ C/O Dr. M.D. Khobragade, Plot No. 238, Kukde Lay Out, Near Pawar Bhawan Nagpur -440027, \\ Maharashtra, India \\ Email: drsonalibhagat@yahoo.in
}

\begin{abstract}
Background: The stress response to laryngoscopy and intubation can cause tachycardia and hypertension resulting in myocardial ischemia and stroke in vulnerable patients. The objective of our study was to compare the efficacy of bolus dose of Esmolol and Lignocaine (preservative free) in attenuation of haemodynamic stress responses to laryngoscopy and intubation in normotensive patients undergoing general anaesthesia for elective surgical procedure.

Material and Methods: sixty patients of ASA grade I and II undergoing elective surgeries under general anaesthesia were randomly divided into 2 groups. Group I $(n=30)$ : received Inj. Esmolol $(2 \mathrm{mg} / \mathrm{kg}$ of body weight) i.v. 3min before laryngoscopy and intubation, over 30seconds. Group II $(n=30)$ : received Inj. lignocaine $(2 \mathrm{mg} / \mathrm{kg}$ of body weight) i.v. $3 \mathrm{~min}$ before laryngoscopy and intubation, over 30seconds. Anaesthesia was standardized in both the groups and vital parameters were recorded for upto 15 minutes after intubation.
\end{abstract}

Results: There was no statistical significant difference in heart rate, systolic blood pressure, diastolic blood pressure and mean arterial pressure in Esmolol and Lignocaine group in the study period except fall in heart rate and systolic blood pressure after administration of intravenous Esmolol $(p<0.05)$.

Conclusion: Esmolol and Lignocaine are equally effective in attenuation of stress response to laryngoscopy and intubation.

Keywords: Esmolol, Lignocaine, Stress response, Intubation, Laryngoscopy. 


\section{Introduction}

Stress response under anesthesia has been universally recognized phenomenon which may be in the form of endocrine or autonomic disturbance. The pressure response to laryngoscopy and endotracheal intubation in the form of tachycardia, hypertension and arrhythmias may be potentially dangerous in vulnerable individuals. These changes are the maximum at 1 minute after intubation and last for 5-10 $\mathrm{min}^{(1)}$. There is substantial evidence that, laryngoscopy and intubation is accompanied by a considerable increase in heart rate and arterial blood pressure. These changes are usually of short duration and well-tolerated by the patients in the absence of cardiovascular disease or disturbed intracranial pressure homeostasis. In these conditions, an increase in blood pressure may lead to complications, including arrhythmias, myocardial ischemia, increase in intracranial pressure and rupture of cerebral aneurysms ${ }^{(2,7)}$. Various methods with use of drugs for attenuation of response to laryngoscopy and intubation are still in search from the date of its recognition. Several studies have been made in order to attenuate these haemodynamic response to laryngoscopy and intubation. Many drugs also have been used for blunting of haemodynamic response, the search for ideal drug with minimal adverse effects continues.

Esmolol is an ultra-short acting $\beta-1$ adrenergic blocker. It has predominant effect on $\beta$-receptors and possesses no significant membrane stabilizing activity. It has rapid onset and a short duration of $\operatorname{action}^{(11)}$.

Lignocaine is an antiarrhythmic medication of the class $1 \mathrm{~b}$ type. Lignocaine works by blocking sodium channels and thus decreasing the rate of contractions of the heart ${ }^{(12)}$. Control of hemodynamic response to endotracheal intubation is important to reduce adverse cardiovascular effects is stressed by various studies. The objective of this study was to compare the efficacy of esmolol and lignocaine in attenuating the pressure response accompanying laryngoscopy and endotracheal intubation and to look for any adverse effects of these drugs.

\section{Study Design}

A prospective, double blind randomized control study was undertaken in tertiary care hospital on 60 ASA I and II patients of age group 18-60 years of either sex, scheduled for elective surgical procedure under general anaesthesia after getting approval from institutional ethical committee. All the patients in the study were clearly explained about the purpose and nature of the study in the language they could understand. They were included in the study only after obtaining a written informed consent.

Inclusion criteria: American Society of Anesthesiologist (ASA) Grade I \& II, Age between 18-50 years, Weight between $40-80 \mathrm{~kg}$ with airway of modified Mallampati Grade I and II were included in the study.

Exclusion criteria: Patients refusal, American Society of Anesthesiologist (ASA) Grade III \& IV, History of seizure disorders, History of cardiovascular diseases like arrhythmias, hypertension, ischaemic heart disease, valvular heart disease, pregnancy, bronchial asthma, HR< $60 / \mathrm{min}, \mathrm{SBP}<100 \mathrm{~mm} \mathrm{Hg}$ suspected difficult airway and Modified Mallampatti Grade III and IV were excluded from the study. Patients who had Cormark Lehane Score III and above and also those in whom duration of laryngoscopy lasted for more than 15 seconds and more were excluded from the study.

\section{Method}

Detailed pre-anaesthetic evaluation of the patients was performed by an anaesthesiologist a day before the surgery. 60 patients satisfying the inclusion and exclusion criteria were included in the study.

Preliminary Investigations were done in the form of; Complete blood count, Random blood sugar, Bleeding time, Clotting time, Coagulation profile, Liver function tests, Kidney function tests, Electrocardiography (ECG), Chest x ray postero- 
anterior (PA) view were noted, Specialized investigation according to the patients for further evaluation if required.

All patients were kept nil by mouth for $8 \mathrm{hrs}$.

All patients were given overnight sedation in the form of Tab. Alprazolam $0.5 \mathrm{mg}$ orally on the night before and 2 hours before surgery.

In operation theatre, multipara monitoring device with ECG, pulse rate, non invasive blood pressure, SPO2 was attached to the patient and baseline parameters were noted. Patients were prehydrated with intravenous Ringer lactate after establishing intravenous line with $18 \mathrm{G}$ cannula. Thereafter, intravenous fluids were calculated and given as per body weight and operative loss. Patients also received Inj. Ranitidine $50 \mathrm{mg}$ and Inj.Ondansetron $4 \mathrm{mg}$ IV slowly as a premedication along with IV midazolam $0.05 \mathrm{mg} / \mathrm{kg}$. Now the patients were randomly divided by computer generated numbers into two groups.

Group I $(\boldsymbol{n}=\mathbf{3 0})$ : received Inj. Esmolol $(2 \mathrm{mg} / \mathrm{kg}$ of bodyweight) i.v. 3 min before laryngoscopy and intubation, over 30 seconds.

Group II $(\boldsymbol{n}=\mathbf{3 0})$ : received Inj. Lignocaine (preservative free $2 \mathrm{mg} / \mathrm{kg}$ of body weight) ) i.v. $3 \mathrm{~min}$ before laryngoscopy and intubation, over 30 seconds.

After preoxygenation for 3-5 minutes with $100 \%$ oxygen, patients were induced with Inj. Propofol $2 \mathrm{mg} / \mathrm{kg}$ and Inj. Vecuronium $0.1 \mathrm{mg} / \mathrm{kg}$ after confirming a lack of response to voice and a loss of the eyelid reflex. Patients were ventilated with oxygen: nitrous oxide (50:50) and sevoflurane 1\% for 3 minutes. Endotracheal intubation was performed by the experienced anaesthesiologist with appropriate size endotracheal tube. Anaesthesia was maintained with controlled ventilation with nitrous oxide and oxygen (60:40) with sevoflurane $2 \%$ with intermittent bolus doses of inj. Vecuronium as muscle relaxant. No surgical stimulation was allowed for 10 minutes after intubation.

Cardiorespiratory parameters (pulse rate, respiratory rate, noninvasive blood pressure,
SPO2, ETCO2) were monitored continuously. Recordings were made till the completion of surgery. At the end of surgery, the residual neuromuscular block was antagonized with neostigmine $(0.05 \mathrm{mg} / \mathrm{kg})$ and glycopyrrolate $(0.01 \mathrm{mg} / \mathrm{kg}) \mathrm{I} . \mathrm{V}$. and extubation was performed when respiration was adequate and patient was able to obey verbal commands with complete return of muscle power.

Intraoperatively and postoperatively, bradycardia (heart rate $<60$ beats per minute) was to be treated with $0.3 \mathrm{mg}$ of injection atropine and hypotension (systolic blood pressure falling more than $20 \%$ basal value or less than $80 \mathrm{~mm} \mathrm{Hg}$ ) with $3-6 \mathrm{mg}$ injection mephenteramine as a bolus.

\section{Statistical Analysis}

The heart rate, systolic blood pressure, diastolic blood pressure and mean rterial pressure were monitored before induction, after study drug, during induction, after laryngoscopy and intubation and thereafter till succeeding 15 minutes. Data were collected, tabulated, coded then analyzed using SPSS ® computer software version 20.0.

- Numerical variables were presented as mean \& standard deviation (SD).

- As regard numerical variables; unpaired student $-\mathrm{t}$ test was done.

- p value

\begin{tabular}{|l|l|}
\hline$>0.05$ & Non Significant \\
\hline$<0.05$ & Significant \\
\hline$<0.001$ & Highly Significant \\
\hline
\end{tabular}

\section{Observations and Results}

The demographic data were comparable in both the groups and are given in table 1 and 2. 
Table No. 1. Demographic Characteristics

\begin{tabular}{|l|c|c|c|c|}
\hline \multicolumn{2}{|l|}{ Demographic character } & group E $(\mathbf{n}=\mathbf{3 0})$ & group L(n=30) & \multirow{2}{*}{ P value } \\
\hline \multirow{2}{*}{ Age (years) } & Mean \pm S.D. & $30.57 \pm 8.23$ & $30.77 \pm 8.09$ & \multirow{2}{*}{$0.919(\mathrm{NS})$} \\
\cline { 2 - 4 } & Range & $18-45$ & $18-45$ & \multirow{2}{*}{$0.813(\mathrm{NS})$} \\
\hline \multirow{2}{*}{ Weight $(\mathrm{kg})$} & Mean \pm S.D. & $55.63 \pm 7.63$ & $55.23 \pm 6.40$ & \\
\cline { 2 - 4 } & Range & $45-75$ & $35-68$ & \multirow{2}{*}{} \\
\hline \multirow{2}{*}{ ASA classification } & Class I & 30 & 0 & \\
\cline { 2 - 4 } & Class II & 0 & 0 & \\
\hline
\end{tabular}

Test applied- student unpaired t-test

Table No. 2: Gender Incidence

\begin{tabular}{|l|c|c|}
\hline Gender & group E(n=30) & group L(n=30) \\
\hline Male (\%) & $26(86.66 \%)$ & $27(90.0 \%)$ \\
\hline Female (\%) & $4(13.33 \%)$ & $3(10.0 \%)$ \\
\hline Total (\%) & $30(100 \%)$ & $30(100 \%)$ \\
\hline
\end{tabular}

Table No.3: Mean $( \pm$ SD) Pulse Rate Alterations In Group I (Esmolol)

\begin{tabular}{|c|c|c|c|c|c|c|c|c|c|c|c|}
\hline $\begin{array}{l}\text { PULSE } \\
\text { RATE }\end{array}$ & $\begin{array}{c}\text { PR } \\
\text { BASAL }\end{array}$ & $\begin{array}{c}\text { AFTER } \\
\text { DRUG }\end{array}$ & $\begin{array}{c}\mathrm{AF} \\
\text { INDUC } \\
\text { TION }\end{array}$ & $\begin{array}{c}\text { AFT } \\
\text { INUBA } \\
\text { TION }\end{array}$ & $1 \mathrm{MIN}$ & $2 \mathrm{MIN}$ & 3 MIN & 4 MIN & $5 \mathrm{MIN}$ & $10 \mathrm{MIN}$ & $15 \mathrm{MIN}$ \\
\hline $\begin{array}{l}\text { ESMO } \\
\text { LOL }\end{array}$ & $\begin{array}{c}79.00 \pm 7 \\
.241 \\
\end{array}$ & $\begin{array}{c}72.13 \pm 5 \\
.866\end{array}$ & $\begin{array}{c}77.67 \pm 1 \\
0.65\end{array}$ & $\begin{array}{c}81.60 \pm 7 . \\
241\end{array}$ & $\begin{array}{c}81.07 \pm 8 \\
.803\end{array}$ & $\begin{array}{c}82.33 \pm 5 \\
.627\end{array}$ & $\begin{array}{c}81.27 \pm \\
6.80\end{array}$ & $\begin{array}{c}81.07 \pm 9 \\
.091\end{array}$ & $\begin{array}{c}81.93 \pm 6 \\
.724 \\
\end{array}$ & $\begin{array}{c}82.07 \pm 6 \\
.053\end{array}$ & $\begin{array}{c}82.33 \pm 6 \\
.945\end{array}$ \\
\hline $\begin{array}{l}\text { P- } \\
\text { VALU } \\
\text { E }\end{array}$ & & $\begin{array}{c}\mathbf{0 . 0 1 6} \\
(\mathrm{S})\end{array}$ & $\begin{array}{c}0.710 \\
\text { (NS) }\end{array}$ & $\begin{array}{c}0.421 \\
(\mathrm{NS})\end{array}$ & $\begin{array}{c}0.503 \\
(\mathrm{NS})\end{array}$ & $\begin{array}{c}0.222 \\
(\mathrm{NS})\end{array}$ & $\begin{array}{c}0.484 \\
(\mathrm{NS})\end{array}$ & $\begin{array}{c}0.485 \\
(\mathrm{NS})\end{array}$ & $\begin{array}{c}0.340 \\
(\mathrm{NS})\end{array}$ & $\begin{array}{c}0.299(\mathrm{~N} \\
\mathrm{S})\end{array}$ & $\begin{array}{c}0.312 \\
\text { (NS) }\end{array}$ \\
\hline
\end{tabular}

There is significant fall in heart rate after administration of i.v. Esmolol. Thereafter, there is no significant change in heart rate in the study period. Fall in the heart rate was never $<60$ beats/ min requiring Inj. Atropine for the treatment.

Table No.4 : Mean $( \pm$ SD) Pulse Rate Alterations in Group II (Lignocaine)

\begin{tabular}{|l|c|c|c|c|c|c|c|c|c|c|c|}
\hline $\begin{array}{l}\text { PULSE } \\
\text { RATE }\end{array}$ & $\begin{array}{c}\text { PR } \\
\text { BASA } \\
\text { L }\end{array}$ & $\begin{array}{c}\text { AFTER } \\
\text { DRUG }\end{array}$ & $\begin{array}{c}\text { AF } \\
\text { INDU } \\
\text { CTION }\end{array}$ & $\begin{array}{c}\text { AFT } \\
\text { INTUBA } \\
\text { TION }\end{array}$ & 1 MIN & 2 MIN & 3 MIN & 4 MIN & 5 MIN & 10 MIN & 15 MIN \\
\hline LIGNOC & $77.80 \pm$ & $79.07 \pm$ & $76.80 \pm$ & $80.13 \pm 8$. & $78.67 \pm$ & $80.60 \pm$ & $78.67 \pm$ & $79.33 \pm$ & $79.80 \pm$ & $79.47 \pm$ & $80.60 \pm$ \\
AINE & 8.026 & 7.015 & 6.527 & 305 & 5.381 & 5.110 & 5.273 & 5.024 & 4.617 & 4.627 & 6.127 \\
\hline P- & & 0.577 & 0.705 & 0.442 & 0.739 & 0.232 & 0.709 & 0.491 & 0.295 & 0.423 & 0.304 \\
VALUE & & (NS) & (NS) & (NS) & (NS) & (NS) & (NS) & (NS) & (NS) & (NS) & (NS) \\
\hline
\end{tabular}

There is no significant change in heart rate in i.v. Lignocaine group in the study period.

Table No.5: Comparison of Mean Pulse Rate Changes between the Groups

\begin{tabular}{|l|c|c|c|c|c|c|c|c|c|c|c|}
\hline $\begin{array}{l}\text { PULSE } \\
\text { RATE }\end{array}$ & $\begin{array}{c}\text { PR } \\
\text { BASA } \\
\text { L }\end{array}$ & $\begin{array}{c}\text { AFTER } \\
\text { DRUG }\end{array}$ & $\begin{array}{c}\text { AF } \\
\text { INDU } \\
\text { CTION }\end{array}$ & $\begin{array}{c}\text { AFT } \\
\text { INTUBA } \\
\text { TION }\end{array}$ & 1 MIN & 2 MIN & 3 MIN & 4 MIN & 5 MIN & 10 MIN & 15 MIN \\
\hline ESMOL & $79.00 \pm$ & $72.13 \pm$ & $77.67 \pm$ & $81.60 \pm 7$. & $81.07 \pm$ & $82.33 \pm$ & $81.27 \pm$ & $81.07 \pm$ & $81.93 \pm$ & $82.07 \pm$ & $82.33 \pm$ \\
OL & 7.241 & 5.866 & 10.65 & 241 & 8.803 & 5.627 & 6.80 & 9.091 & 6.724 & 6.053 & 6.945 \\
\hline LIGNOC & $77.80 \pm$ & $79.07 \pm$ & $76.80 \pm$ & $80.13 \pm 8$. & $78.67 \pm$ & $80.60 \pm$ & $78.67 \pm$ & $79.33 \pm$ & $79.80 \pm$ & $79.47 \pm$ & $80.60 \pm$ \\
AINE & 8.026 & 7.015 & 6.527 & 305 & 5.381 & 5.110 & 5.273 & 5.024 & 4.617 & 4.627 & 6.127 \\
\hline P & 0.626 & $\mathbf{0 . 0 0 7}$ & 0.790 & 0.645 & 0.375 & 0.385 & 0.252 & 0.523 & 0.320 & 0.197 & 0.475 \\
VALUE & $(\mathrm{NS})$ & $\mathbf{( S )}$ & $(\mathrm{NS})$ & $(\mathrm{NS})$ & $(\mathrm{NS})$ & $(\mathrm{NS})$ & $(\mathrm{NS})$ & $(\mathrm{NS})$ & $(\mathrm{NS})$ & $(\mathrm{NS})$ & (NS) \\
\hline
\end{tabular}

On intergroup comparison, there was no statistically significant difference in pulse rate till 15 minutes except the pulse rate in Esmolol group was significantly lower than Lignocaine after drug administration. 
Table No.6: Mean $( \pm$ SD) Systolic Blood Pressure Alterations In Group I (Esmolol)

\begin{tabular}{|l|c|c|c|c|c|c|c|c|c|c|c|}
$\begin{array}{l}\text { SYSTO } \\
\text { LIC BP }\end{array}$ & $\begin{array}{c}\text { SBP } \\
\text { BASA } \\
\text { L }\end{array}$ & $\begin{array}{c}\text { AFTER } \\
\text { DRUG }\end{array}$ & $\begin{array}{c}\text { AF } \\
\text { INDU } \\
\text { CTION }\end{array}$ & $\begin{array}{c}\text { AFT } \\
\text { INTUBA } \\
\text { TION }\end{array}$ & 1 MIN & 2 MIN & 3 MIN & 4 MIN & 5 MIN & 10 MIN & 15 MIN \\
\hline ESMOL & 123.27 & $\mathbf{1 0 6 . 0} \pm$ & $121.27 \pm$ & $127 \pm 9.07$ & $126.40 \pm$ & $121.87 \pm$ & 120.27 & 126.60 & 121.33 & 121.60 & 121.07 \\
OL & \pm 6.81 & $\mathbf{2 . 9 0 3}$ & 23.57 & 1 & 16.51 & 16.59 & \pm 9.26 & \pm 7.99 & \pm 8.52 & \pm 9.82 & \pm 9.22 \\
\hline P- & & $\mathbf{0 . 0 0 0}$ & 0.772 & 0.210 & 0.484 & 0.754 & 0.280 & 0.160 & 0.485 & 0.529 & 0.535 \\
VALUE & & $\mathbf{( H S )}$ & (NS) & (NS) & (NS) & (NS) & (NS) & (NS) & (NS) & (NS) & (NS) \\
\hline
\end{tabular}

In our study, there was significant fall in systolic blood pressure after administration of i.v. remained near the basal values till 15 minutes. Esmolol. Thereafter systolic blood pressure

Fall in the SBP did not required any treatment.

Table No.7: Mean ( \pm SD) Systolic Blood Pressure Alterations In Group II (Lignocaine)

\begin{tabular}{|l|c|c|c|c|c|c|c|c|c|c|c|}
\hline $\begin{array}{l}\text { SYSTOL } \\
\text { IC BP }\end{array}$ & $\begin{array}{c}\text { SBP } \\
\text { BASA } \\
\text { L }\end{array}$ & $\begin{array}{c}\text { AFTER } \\
\text { DRUG }\end{array}$ & $\begin{array}{c}\text { AF } \\
\text { INDU } \\
\text { CTION }\end{array}$ & $\begin{array}{c}\text { AFT } \\
\text { INTUBA } \\
\text { TION }\end{array}$ & 1 MIN & 2 MIN & 3 MIN & 4 MIN & 5 MIN & 10 MIN & 15 MIN \\
\hline LIGNOC & 121.13 & 116.07 & 120.13 & $126.00 \pm 8$ & $125.73 \pm$ & 120.20 & 121.60 & 123.87 & 120.13 & 119.47 & 119.67 \\
AINE & \pm 5.89 & \pm 8.31 & \pm 9.22 & .00 & 14.97 & \pm 9.00 & \pm 5.69 & \pm 7.87 & \pm 8.06 & \pm 8.33 & \pm 7.87 \\
\hline P- & & 0.067 & 0.754 & 0.065 & 0.313 & 0.743 & 0.816 & 0.296 & 0.602 & 0.464 & 0.614 \\
VALUE & & (NS) & (NS) & (NS) & (NS) & (NS) & (NS) & (NS) & (NS) & (NS) & (NS) \\
\hline
\end{tabular}

In our study, there was statistically insignificant change in systolic blood pressure in i.v. Lignocaine group.

Table No.8: Comparison of Mean Systolic Blood Pressure Changes between the Groups

\begin{tabular}{|c|c|c|c|c|c|c|c|c|c|c|c|}
\hline $\begin{array}{l}\text { SYSTOL } \\
\text { IC BP }\end{array}$ & $\begin{array}{c}\text { SBP } \\
\text { BASA } \\
\text { L }\end{array}$ & $\begin{array}{l}\text { AFTER } \\
\text { DRUG }\end{array}$ & $\begin{array}{c}\mathrm{AF} \\
\text { INDU } \\
\text { CTION }\end{array}$ & $\begin{array}{c}\text { AFT } \\
\text { INTUBA } \\
\text { TION }\end{array}$ & $1 \mathrm{MIN}$ & $2 \mathrm{MIN}$ & $3 \mathrm{MIN}$ & $4 \mathrm{MIN}$ & $5 \mathrm{MIN}$ & $\begin{array}{c}10 \\
\text { MIN }\end{array}$ & $\begin{array}{c}15 \\
\text { MIN }\end{array}$ \\
\hline $\begin{array}{l}\text { ESMOL } \\
\text { OL }\end{array}$ & $\begin{array}{c}123.27 \\
\pm 6.81\end{array}$ & $\begin{array}{c}106.0 \pm 2 \\
.903\end{array}$ & & $\begin{array}{c}127 \pm 9.07 \\
1\end{array}$ & & & & & & & \\
\hline $\begin{array}{l}\text { LIGNOC } \\
\text { AINE }\end{array}$ & $\begin{array}{l}121.13 \\
\pm 5.89 \\
\end{array}$ & $\begin{array}{c}116.07 \pm \\
8.311 \\
\end{array}$ & $\begin{array}{c}120.13 \pm \\
9.22 \\
\end{array}$ & $\begin{array}{c}126.00 \pm 8 \\
.00\end{array}$ & & $\begin{array}{c}120.20 \pm \\
9.00 \\
\end{array}$ & & & & $\begin{array}{l}119.47 \\
\pm 8.33 \\
\end{array}$ & $\begin{array}{l}119.67 \\
\pm 7.87\end{array}$ \\
\hline $\begin{array}{l}\mathrm{P} \\
\text { VALUE }\end{array}$ & $\begin{array}{l}0.366^{\prime} \\
\text { (NS) }\end{array}$ & $\begin{array}{c}\mathbf{0 . 0 0 0} \\
(\mathrm{HS})\end{array}$ & $\begin{array}{c}0.864 \\
\text { (NS) }\end{array}$ & $\begin{array}{l}0.751 \\
\text { (NS) }\end{array}$ & $\begin{array}{c}0.909 \\
(\mathrm{NS})\end{array}$ & $\begin{array}{l}0.735 \\
\text { (NS) }\end{array}$ & $\begin{array}{l}0.638 \\
\text { (NS) }\end{array}$ & $\begin{array}{l}0.353 \\
\text { (NS) }\end{array}$ & $\begin{array}{l}0.695 \\
\text { (NS) }\end{array}$ & $\begin{array}{l}0.526 \\
\text { (NS) }\end{array}$ & $\begin{array}{c}0.658 \\
\text { (NS) }\end{array}$ \\
\hline
\end{tabular}

On intergroup comparison, there was no pressure in Esmolol group was significantly lower statistically significant difference in systolic blood than Lignocaine after drug administration.

pressure till 15 minutes except the systolic blood

Table No.9: Mean ( \pm SD) Diastolic Blood Pressure Alterations In Group I (Esmolol)

\begin{tabular}{|l|c|c|c|c|c|c|c|c|c|c|c|}
\hline $\begin{array}{l}\text { DIASTO } \\
\text { LIC BP }\end{array}$ & $\begin{array}{c}\text { DBP } \\
\text { BASA } \\
\text { L }\end{array}$ & $\begin{array}{c}\text { AFTER } \\
\text { DRUG }\end{array}$ & $\begin{array}{c}\text { AF } \\
\text { INDU } \\
\text { CTION }\end{array}$ & $\begin{array}{c}\text { AFT } \\
\text { INTUBA } \\
\text { TION }\end{array}$ & 1 MIN & 2 MIN & 3 MIN & 4 MIN & MIN & 10 & 15 \\
\hline ESMOL & $75.13 \pm$ & $79.60 \pm$ & $69.40 \pm$ & $70.14 \pm 8.8$ & $69.25 \pm$ & $76.20 \pm 1$ & $76.93 \pm$ & $75.40 \pm$ & $72.33 \pm 1$ & $70.07 \pm$ & $74.13 \pm$ \\
OL & 7.43 & 7.80 & 8.71 & 1 & 6.88 & 6.32 & 9.92 & 6.29 & 0.06 & 9.66 & 7.05 \\
\hline P- & & 0.082 & 0.056 & 0.265 & 0.105 & 0.819 & 0.617 & 0.916 & 0.475 & 0.190 & 0.522 \\
VALUE & & (NS) & (NS) & (NS) & (NS) & (NS) & (NS) & (NS) & (NS) & (NS) & (NS) \\
\hline
\end{tabular}

There was statistically insignificant change in diastolic blood pressure in i.v. Esmolol group.

Table No.10: Mean $( \pm$ SD) Diastolic Blood Pressure Alterations in Group II (Lignocaine)

\begin{tabular}{|l|c|c|c|c|c|c|c|c|c|c|c|}
\hline $\begin{array}{l}\text { DIASTOL } \\
\text { IC BP }\end{array}$ & $\begin{array}{c}\text { DBP } \\
\text { BASA } \\
\text { L }\end{array}$ & $\begin{array}{c}\text { AFTER } \\
\text { DRUG }\end{array}$ & $\begin{array}{c}\text { AF } \\
\text { INDU } \\
\text { CTION }\end{array}$ & $\begin{array}{c}\text { AFT } \\
\text { INTUBA } \\
\text { TION }\end{array}$ & 1 MIN & 2 MIN & 3 MIN & 4 & 5 MIN & 10 & 15 \\
LIGNOC & $74.33 \pm$ & $77.00 \pm$ & $71.33 \pm$ & $73.36 \pm 6.1$ & $72.92 \pm$ & $75.80 \pm$ & $77.00 \pm$ & $74.93 \pm$ & $71.53 \pm$ & $71.60 \pm$ & $72.60 \pm$ \\
AINE & 7.43 & 7.17 & 6.09 & 1 & 4.01 & 9.53 & 6.40 & 4.73 & 7.53 & 8.39 & 5.36 \\
\hline P-VALUE & & 0.270 & 0.145 & 0.793 & 0.312 & 0.610 & 0.234 & 0.718 & 0.286 & 0.285 & 0.288 \\
& & (NS) & (NS) & (NS) & (NS) & (NS) & (NS) & (NS) & (NS) & (NS) & (NS) \\
\hline
\end{tabular}

There was statistically insignificant change in diastolic blood pressure in i.v. Lignocaine group. 
Table No.11: Comparison of Mean Diastolic Blood Pressure Changes between the Groups

\begin{tabular}{|l|c|c|c|c|c|c|c|c|c|c|c|}
\hline $\begin{array}{l}\text { DIASTO } \\
\text { LIC BP }\end{array}$ & $\begin{array}{c}\text { DBP } \\
\text { BASA }\end{array}$ & $\begin{array}{c}\text { AFTE } \\
\text { R }\end{array}$ & $\begin{array}{c}\text { AF } \\
\text { DRUG }\end{array}$ & $\begin{array}{c}\text { AFT } \\
\text { CTION }\end{array}$ & $\begin{array}{c}1 \text { MIN } \\
\text { INTBA }\end{array}$ & 2 MIN & 3 MIN & 4 & 5 MIN & 10 & 15 \\
\hline ESMOLO & $75.13 \pm$ & $79.60 \pm$ & $69.40 \pm$ & $70.14 \pm 8.8$ & $69.25 \pm$ & $76.20 \pm 1$ & $76.93 \pm$ & $75.40 \pm$ & $72.33 \pm 1$ & $70.07 \pm$ & $74.13 \pm$ \\
L & 7.43 & 7.80 & 8.71 & 1 & 6.88 & 6.32 & 9.92 & 6.29 & 0.06 & 9.66 & 7.05 \\
\hline LIGNOC & $74.33 \pm$ & $77.00 \pm$ & $71.33 \pm$ & $73.36 \pm 6.1$ & $72.92 \pm$ & $75.80 \pm 9$ & $77.00 \pm$ & $74.93 \pm$ & $71.53 \pm 7$ & $71.60 \pm$ & $72.60 \pm$ \\
AINE & 7.43 & 7.17 & 6.09 & 1 & 4.01 & .53 & 6.40 & 4.73 & .53 & 8.39 & 5.36 \\
\hline P- & 0.711 & 0.350 & 0.487 & 0.272 & 0.125 & 0.935 & 0.983 & 0.820 & 0.807 & 0.646 & 0.508 \\
VALUE & (NS) & (NS) & (NS) & (NS) & (NS) & (NS) & (NS) & (NS) & (NS) & (NS) & (NS) \\
\hline
\end{tabular}

On intergroup comparison, there was statistically insignificant change in diastolic blood pressure in both the groups.

Table No.12: Mean ( \pm SD) Arterial Pressure Alterations in Group I (Esmolol)

\begin{tabular}{|l|c|c|c|c|c|c|c|c|c|c|c|}
\hline $\begin{array}{l}\text { MEAN } \\
\text { ARTER } \\
\text { IAL BP }\end{array}$ & $\begin{array}{c}\text { MAP } \\
\text { BASA }\end{array}$ & $\begin{array}{c}\text { AFTER } \\
\text { DRUG }\end{array}$ & $\begin{array}{c}\text { AF } \\
\text { INDU } \\
\text { CTION }\end{array}$ & $\begin{array}{c}\text { AFT } \\
\text { INTUBA } \\
\text { TION }\end{array}$ & 1 MIN & 2 MIN & 3 MIN & 4 MIN & 5 MIN & 10 & 15 \\
\hline ESMOL & $91.18 \pm$ & $88.40 \pm$ & $96.33 \pm 1$ & $86.711 \pm 4$ & $81.15 \pm 2$ & $91.42 \pm 1$ & $91.38 \pm$ & $92.47 \pm$ & $86.20 \pm$ & $87.24 \pm$ & $89.78 \pm$ \\
OL & 6.85 & 5.17 & 7.97 & 77 & 3.17 & 5.28 & 8.34 & 5.49 & 6.99 & 6.44 & 5.36 \\
\hline P- & & 0.176 & 0.354 & 0.053 & 0.206 & 0.955 & 0.946 & 0.515 & 0.088 & 0.173 & 0.451 \\
VALUE & & (NS) & (NS) & (NS) & (NS) & (NS) & (NS) & (NS) & (NS) & (NS) & (NS) \\
\hline
\end{tabular}

There was statistically insignificant change in mean arterial pressure in i.v. Esmolol group.

Table No.13: Mean $( \pm$ SD) Arterial Pressure Alterations in Group II (Lignocaine)

\begin{tabular}{|c|c|c|c|c|c|c|c|c|c|c|c|}
\hline $\begin{array}{l}\text { MEAN } \\
\text { ARTERI } \\
\text { AL BP }\end{array}$ & $\begin{array}{c}\text { MAP } \\
\text { BASA } \\
\text { L }\end{array}$ & $\begin{array}{l}\text { AFTE } \\
\text { R } \\
\text { DRUG }\end{array}$ & $\begin{array}{c}\text { AF } \\
\text { INDU } \\
\text { CTION }\end{array}$ & $\begin{array}{c}\text { AFT } \\
\text { INTUBA } \\
\text { TION }\end{array}$ & $1 \mathrm{MIN}$ & $2 \mathrm{MIN}$ & $3 \mathrm{MIN}$ & $4 \mathrm{MIN}$ & $5 \mathrm{MIN}$ & $\begin{array}{c}10 \\
\text { MIN }\end{array}$ & $\begin{array}{c}15 \\
\text { MIN }\end{array}$ \\
\hline $\begin{array}{l}\text { LIGNOC } \\
\text { AINE }\end{array}$ & $\begin{array}{c}89.93 \pm \\
3.75\end{array}$ & $\begin{array}{c}90.02 \pm \\
5.79\end{array}$ & $\begin{array}{c}94.98 \pm \\
8.61\end{array}$ & $\begin{array}{c}87.60 \pm 3.8 \\
7\end{array}$ & $\begin{array}{c}83.35 \pm 2 \\
3.53\end{array}$ & $\begin{array}{c}90.60 \pm \\
7.11\end{array}$ & $\begin{array}{c}91.87 \pm \\
4.52\end{array}$ & $\begin{array}{c}91.24 \pm \\
4.22\end{array}$ & $\begin{array}{c}86.20 \pm \\
5.69\end{array}$ & $\begin{array}{c}87.55 \pm \\
6.06\end{array}$ & $\begin{array}{c}88.29 \pm \\
4.27\end{array}$ \\
\hline $\begin{array}{l}\text { P- } \\
\text { VALUE }\end{array}$ & & $\begin{array}{l}0.964 \\
(\mathrm{NS})\end{array}$ & $\begin{array}{l}0.065 \\
\text { (NS) }\end{array}$ & $\begin{array}{c}0.076 \\
(\mathrm{NS})\end{array}$ & $\begin{array}{c}0.339 \\
\text { (NS) }\end{array}$ & $\begin{array}{l}0.771 \\
(\mathrm{NS})\end{array}$ & $\begin{array}{l}0279 . \\
(\mathrm{NS})\end{array}$ & $\begin{array}{c}0.359 \\
\text { (NS) } \\
\end{array}$ & $\begin{array}{c}0.104 \\
(\mathrm{NS}) \\
\end{array}$ & $\begin{array}{c}0.219 \\
(\mathrm{NS}) \\
\end{array}$ & $\begin{array}{c}0.323 \\
(\mathrm{NS}) \\
\end{array}$ \\
\hline
\end{tabular}

There was statistically insignificant change in mean arterial pressure in i.v. Lignocaine group.

Table No.14: Comparison of Mean Arterial Pressure Changes between the Groups

\begin{tabular}{|l|c|c|c|c|c|c|c|c|c|c|c|}
\hline MEAN & MAP & AFTE & AF & AFT & 1 MIN & 2 MIN & 3 MIN & 4 MIN & 5 MIN & 10 & 15 \\
AL BP & $\begin{array}{c}\text { BASA } \\
\text { L }\end{array}$ & $\begin{array}{c}\text { R } \\
\text { DRUG }\end{array}$ & $\begin{array}{c}\text { INDU } \\
\text { CTION }\end{array}$ & $\begin{array}{c}\text { INTUBA } \\
\text { TION }\end{array}$ & & & & & & MIN & MIN \\
\hline ESMOLO & $91.18 \pm$ & $88.40 \pm$ & $96.33 \pm 1$ & $86.711 \pm 4$ & $81.15 \pm 2$ & $91.42 \pm 1$ & $91.38 \pm$ & $92.47 \pm$ & $86.20 \pm$ & $87.24 \pm$ & $89.78 \pm$ \\
L & 6.85 & 5.17 & 7.97 & 77 & 3.17 & 5.28 & 8.34 & 5.49 & 6.99 & 6.44 & 5.36 \\
\hline LIGNOC & $89.93 \pm$ & $90.02 \pm$ & $94.98 \pm 8$ & $87.60 \pm 3.8$ & $83.35 \pm 2$ & $90.60 \pm 7$ & $91.87 \pm$ & $91.24 \pm$ & $86.20 \pm$ & $87.55 \pm$ & $88.29 \pm$ \\
AINE & 3.75 & 5.79 & .61 & 7 & 3.53 & .11 & 4.52 & 4.22 & 5.69 & 6.06 & 4.27 \\
\hline P- & 0.542 & 0.425 & 0.794 & 0.579 & 0.798 & 0.851 & 0.843 & 0.500 & 1.000 & 0.893 & 0.408 \\
VALUE & (NS) & (NS) & (NS) & (NS) & (NS) & (NS) & (NS) & (NS) & (NS) & (NS) & (NS) \\
\hline
\end{tabular}

On intergroup comparison, there was statistically insignificant change in mean arterial pressure in both the groups.

\section{Perioperative Complication}

None of the patient developed any complications like hypotension, bradycardia, arrhythmias and allergic reaction in perioperative period.

\section{Discussion}

Endotracheal intubation as well as laryngoscopy provides an intense stimulus via vagal and glossopharyngeal afferents that results in a reflex autonomic stimulation manifested in the form of hypertension and tachycardia in adults and adolescents. This autonomic activation may result in bradycardia in infants and small children. Hypertension and bradycardia are usually of short duration; however, they may have consequences in patients with significant cardiac disease. Central nervous system activation as a result of airway management results in increase in electroencephalographic (EEG) activity, increase 
cerebral blood flow and cerebral metabolic rate which may result in an increase in intracranial pressure in patients with decreased intracranial compliance $^{(3)}$. Esmolol is a cardio selective beta adrenergic blocking drug with rapid onset and short elimination half life (9 $\mathrm{min})$ without any significant drug interaction with commonly used anaesthetic drugs. Esmolol decrease the heart rate and force of contraction by blocking of beta adrenergic receptors of the heart, blood vessels, and other organs of the body. Esmolol attenuates the tachycardia and hypertension due to laryngoscopy and intubation by preventing the action of two naturally occurring neurotransmitters epinephrine and nor epinephrine. There have been various studies describing the effects on heart rate and blood pressure during laryngoscopy and intubation of esmolol. Korpinen et $\mathrm{al}^{(5)}$ concluded that bolus Esmolol 2mg/kg 2 min before laryngoscopy and intubation prevented increase in heart rate rather than increase in arterial blood pressure. Singh S et $\mathrm{al}^{(6)}$ justified the usage of higher dose of Esmolol $2 \mathrm{mg} / \mathrm{kg}$ in ghanian population without any hypotension and bradycardia. Suresh kumar singhal et $\mathrm{al}^{(7)}$ reported that bolus intravenous dose of Esmolol $1.5 \mathrm{mg} / \mathrm{kg}$ is safe and more effective in attenuating the hemodynamic response to laryngoscopy and intubation when administered three minutes before intubation. M. Andrew levitt et $\mathrm{al}^{(8)}$ concluded that esmolol $2 \mathrm{mg} / \mathrm{kg}$ and lignocaine $2 \mathrm{mg} / \mathrm{kg}$ have similar efficacies to attenuate hemodynamic response to intubation of patients with isolated head trauma. In our study, Esmolol $2 \mathrm{mg} / \mathrm{kg}$ as a bolus was found to be effective in attenuation of hypertensive stress response as well as tachycardia during laryngoscopy and tracheal intubation till 5 min without any deleterious effect.

Lignocaine attenuates haemodynamic responses during laryngoscopy and intubation due to its direct cardiac depressant effect along with peripheral vasodialatory action. Lignocaine also suppress airway reflexes due to irritation of tracheal mucosa and has analgesic and antiarrhythmic properties. Singh $\mathrm{S}$ et $\mathrm{al}^{(6)}$ found that lignocaine $1.5 \mathrm{mg} / \mathrm{kg}$ and Esmolol $2 \mathrm{mg} / \mathrm{kg}$ are effective in suppressing the haemodynamic response to laryngoscopy and intubation without any deleterious effect. Lev and $\operatorname{Rosen}^{(9)}$ concluded that IV lignocaine $1.5 \mathrm{mg} / \mathrm{kg} 3$ minutes before intubation was optimal for attenuation of sympathoadrenal response to laryngoscopy and intubation. Jain $\mathrm{P}$, vats $\mathrm{A}^{(10)}$ reported that intravenous lignocaine $2 \mathrm{mg} / \mathrm{kg}$ and esmolol 2 $\mathrm{mg} / \mathrm{kg}$ are effective in attenuating the haemodynamic response to laryngoscopy and intubation for about $5 \mathrm{~min}$ without any deleterious effect.

However some studies conducted by Gupta A et $\mathrm{al}^{(11)}$, Kindler et al ${ }^{(12)}$, Miller CD et al ${ }^{(13)}$ and Van den berg et $\mathrm{al}^{(14)}$ disagree the lignocaine' s effect on attenuation of stress response to laryngoscopy and intubation as bolus intravenous dose of $1.5 \mathrm{mg} / \mathrm{kg}$. Hence we modify the dose of intravenous lignocaine as $2 \mathrm{mg} / \mathrm{kg}$. In our study, lignocaine $2 \mathrm{mg} / \mathrm{kg}$ was found to be effective in blunting the haemodynamic response to laryngoscopy and intubation.

\section{Conclusion}

From the present study, based on the results and methodology applied, we conclude that intravenous Lignocaine (preservative free) 2 $\mathrm{mg} / \mathrm{kg}$ and Esmolol $2 \mathrm{mg} / \mathrm{kg}$ are effective in attenuating the haemodynamic stress response to laryngoscopy and tracheal intubation for about 15 minutes without any deleterious effect.

\section{References}

1. King BD, Harris LC jr, Greifenstein FE, Elder JD, Dripps RD. Reflex circulatory responses to direct laryngoscopy and tracheal intubation performed during general anaesthesia. Anesthesiology 1951;12:556-66.

2. Kovac AL. Controlling the hemodynamic response to laryngoscopy and endotracheal intubation. Journal of Clinical Anaesthesia 1996;8:63-79. 
3. Joffe AM, Deem SA: physiologic and pathophysiologic responses to intubation. In Hagberg CA, editor: Benumof's airway management: principles and practice, ed 3, Philadelphia, 2012, Saunders,p184.

4. Stoelting RK. Circulatory changes during direct laryngoscopy and tracheal intubation: influence of duration of laryngoscopy with or without lignocaine. Anaesthesiology 1977;47: 381-84.

5. Korpinen R, Simola M, Saarnivaara L. Effect of esmolol on the hemodynamic and electrocardiographic changes during laryngomicroscopy under propofol- alfentanil anesthesia. Acta Anaesthesiol Belg 1998;49:123-32.

6. Singh S, Laing EF, Owiredu WK, Singh A. Comparison of esmolol and lidocaine for attenuation of cardiovascular stress response to laryngoscopy and endotracheal intubation in a ghanian population.

7. Singhal SK, Malhotra N, kaur K, Dhaiya D. Efficacy of esmolol administration at different time intervals in attenuating hemodynamic response to tracheal intubation. Indian journal of medical sciences 2010; vol64: 468-75.

8. Levitt MA, DO, Dresden GM, AB. The efficacy of esmolol versus lidocaine to attenuate the hemodynamic response to intubation in isolated head trauma patients. Academic emergency medicine 2001;8:19-24.

9. Lev R, Rosen P. Prophylactic lidocaine use preintubation: A review. J Emerg Med 1994;12:499-506.

10. Jain P, Vats A. Comparison of esmolol and lidocaine for blunting of stress response during laryngoscopy and endotracheal intubation. International J of Scientific Study 2017;5:12-17.

11. Gupta A, Wakhloo R, Gupta V, Mehta A, Kapoor BB. Comparison of esmolol and lingocaine for attenuation of cardiovascular stress response to laryngoscopy and endotracheal intubation. J jkscience 2009;11:78-81.
12. Kindler CH, Schumacher PG, Schneider MC, Urwyler A. Effects of intravenous lidocaine and /or esmolol on hemodynamic responses to laryngoscopy and intubation: a double- blind, controlled clinical trial. J Clin Anesth 1996;8:491-6.

13. Miller CD, Warren SJ. Intravenous lignocaine fails to attenuate the cardiovascular response to laryngoscopy and tracheal intubation. Br J Anaesth 1990;65:216-9.

14. Van den Berg AA, Savva D, Honjal MN. Attenuation of the hemodynamic stress responses to noxious stimuli in patients undergoing cataract surgery. A comparison of magnesium sulphate, esmolol, lignocaine, nitroglyserine and placebo given i.v. with induction of anaesthesia. Eur J Anaesthesiol 1997;14:134-47.

15. Bostana H, Eroglu A. Comparison of the clinical efficacies of fentanyl, esmolol and lignocaine in preventing the haemodynamic response to endotracheal intubation and extubation.J Curr Surg 2012;2:24-8.

16. Shroff PP, Mohite SN, Panchal ID. bolus administration of esmolol in controlling the hemodynamic response to tracheal intubation.J Anaesthesiol Clin Pharmacol2004;20:69-72.

17. Savio KH, Tait G, Karkouti K, Wijeysundera D, McCluskey S, Beattie WS. the safety of perioperative esmolol: a systematic review and meta-analysis of randomized controlled trials. Anesth Analg 2011;112:267-81.

18. Abou- Madi MN, Keszler H, Yacoub JM. Cardiovascular reactions to laryngoscopy and intubation following small and large intravenous doses of lignocaine. Can Anaesth Soc J 1977;24:12-19.

19. Wilson IG, Meiklejohn BH, Smith G. Intravenous lignocaine and sympathoadrenal responses to laryngoscopy and intubation. The effect of varying time of injection. Anaesthesia 1991;46:177-80. 